\title{
Malignant peripheral nerve sheath tumor arising from the greater omentum: Case report
}

\author{
Masashi Miguchi, Yuji Takakura*, Hiroyuki Egi, Takao Hinoi, Tomohiro Adachi, Yasuo Kawaguchi, \\ Manabu Shinomura, Masakazu Tokunaga, Masazumi Okajima, Hideki Ohdan
}

\begin{abstract}
Malignant peripheral nerve sheath tumors (MPNSTs) are rare soft tissue tumors that arise from a peripheral nerve or exhibit nerve sheath differentiation. Most of these tumors arise on the trunk, extremities, or head and neck regions; they are very rarely located in the abdominal cavity. The patient was a 71-year-old man who was referred to our hospital for a mass and pain in the right lower abdomen. Abdominal computed tomography revealed a large $(9 \times$ $9 \mathrm{~cm}$ ), well-circumscribed, lobulated, heterogeneously enhanced mass in the pelvis. Exploratory laparotomy revealed a large mass in the greater omentum, and the tumor was completely excised. Histopathological analysis revealed that the tumor was composed of spindle cells with high mitotic activity. On staining the tumor, positive results were obtained for S-100 but negative results were obtained for c-kit, cluster of differentiation (CD)34, $\alpha$ smooth muscle actin, and desmin. These findings strongly supported a diagnosis of MPNST primarily arising from the greater omentum. To the best of our knowledge, this is the first reported case of an MPNST arising from the greater omentum. In this report, we have described the case of a patient with an MPNST arising from the greater omentum and have discussed the clinical characteristics and management of MPNSTs.
\end{abstract}

\section{Background}

Primary solid omental tumors are rare and include various types of tumors such as gastrointestinal stromal tumors (GIST), leiomyosarcomas, hemangiocytomas, fibrosarcomas, leiomyomas, liposarcomas, desmoids tumors, fibromas, mesotheliomas, and myosarcomas [1]. Although the pathological spectrum of primary omental tumors is diverse, no report has yet been published on malignant peripheral nerve sheath tumors (MPNSTs) arising from the greater omentum.

In this report, we describe the extremely rare case of a Japanese man who had an MPNST arising from the greater omentum.

\section{Case presentation}

The patient was a 71-year-old man who was healthy by birth and was admitted to our hospital with pain in the right lower abdomen. Physical examination revealed a large, firm, movable mass in the abdomen. The hematological tests, including those for the serum levels of

\footnotetext{
* Correspondence: ytaka0621@aol.com

Deparment of Gastroenterological Surgery, Hiroshima University Hospital 1-23 Kasumi, Minami-ku, Hiroshima city, Hiroshima 734-8551, Japan
}

tumor markers such as carcinoembryonic antigen (CEA), carbohydrate antigen (CA) 19-9, and CA125, yielded normal results. Abdominal computed tomography $(\mathrm{CT})$ revealed a large (approximately, $9 \times 9 \mathrm{~cm}$ ), well-circumscribed, lobulated mass in the pelvis. The central region of the mass appeared to have low density, while the marginal region was well enhanced in the CT scan (Figure 1A). CT/positron emission tomography (PET) with ${ }^{18} \mathrm{~F}$-fluorodeoxyglucose (FDG) showed a mass with increased FDG accumulation in the right lower abdomen, without any evidence of distant metastasis (Figure 1B). Evaluation of the gastrointestinal tract did not yield any definite results. The origin of the tumor could not be clearly determined.

Exploratory laparotomy was performed under the diagnosis of an intra-abdominal tumor of unknown origin. During laparotomy, it was observed that the tumor arose from the greater omentum and was not connected with the gastrointestinal tract (Figure 2). The tumor was completely excised along with the greater omentum.

Gross pathological examination revealed that the tumor was a whitish-grey oval mass, with a maximum diameter of $9 \mathrm{~cm}$ (Figure 3). Microscopic examination revealed spindle cells arranged in intersecting fascicles 


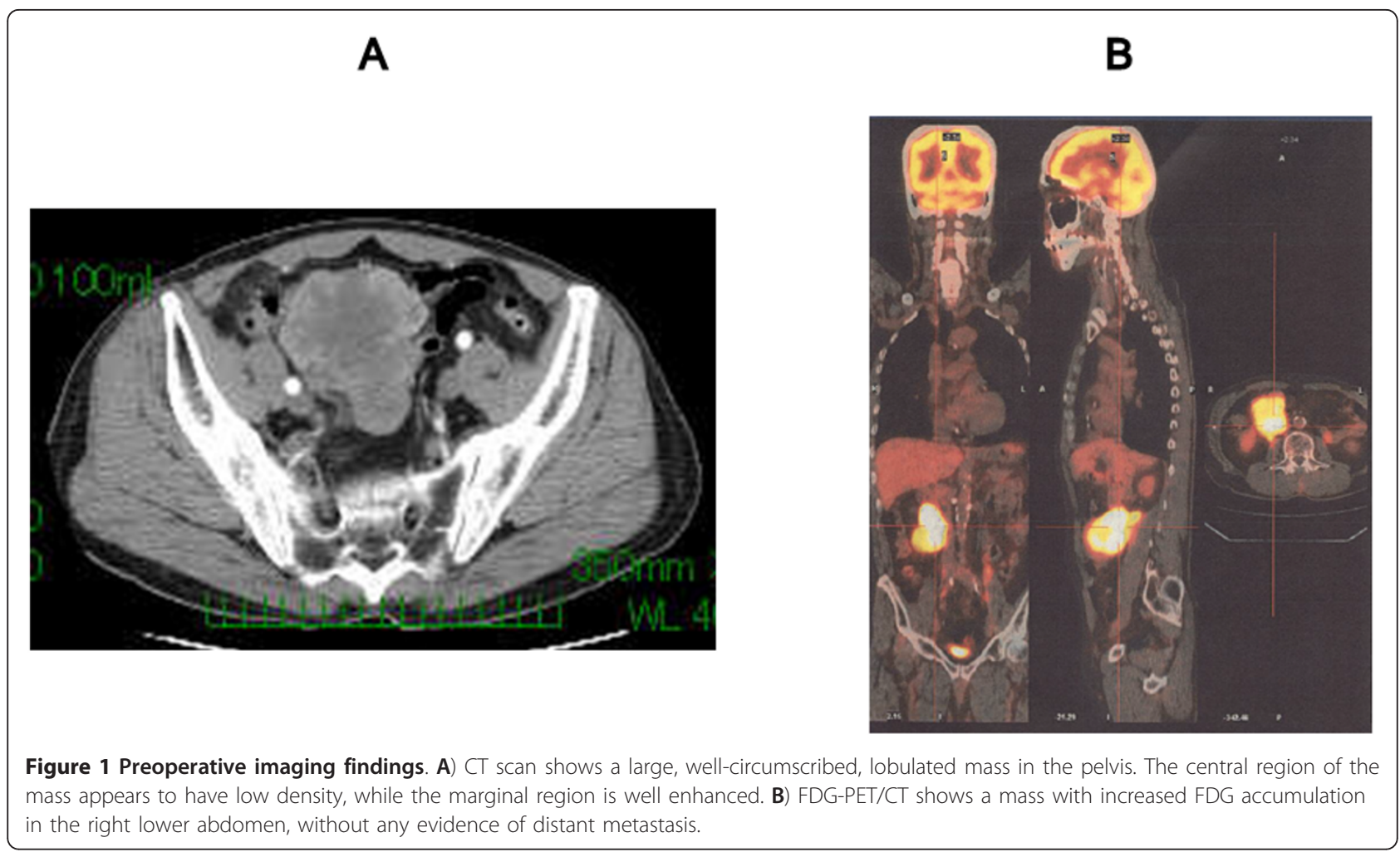

and polygonal cells arranged in sheets grow infiltrating (Figure 4A). The cellular nuclei were polygonal (bulky, roundish, and irregular), and the mitotic activity was 150 mitoses per 50 high-power fields. Coagulative necrosis and myxoid changes were observed in the tumor. Immunohistochemical analysis of the tumor cells yielded positive staining results for S-100 (Figure 4B) but negative results for c-kit, $\alpha$-smooth muscle actin $(\alpha$ SMA), desmin, and cluster of differentiation (CD) 34

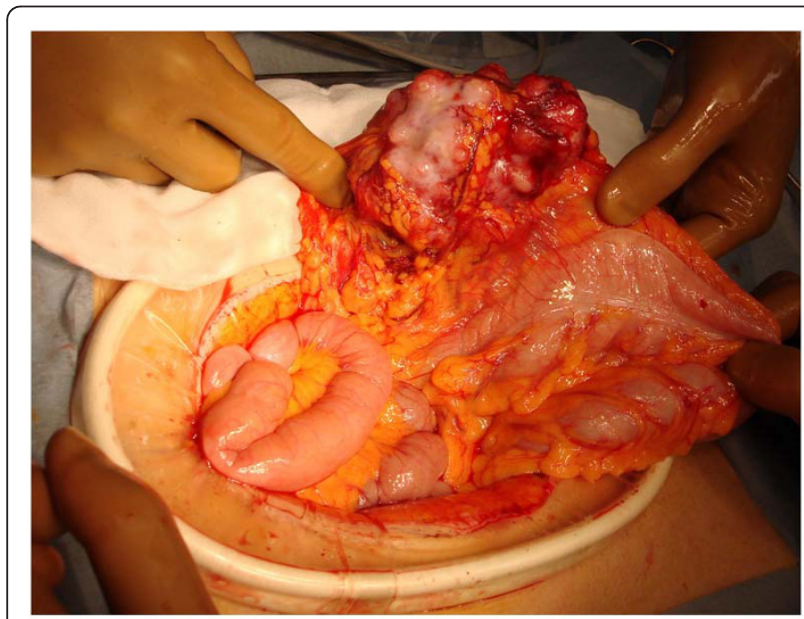

Figure 2 The tumor arises from the greater omentum and is not connected with the gastrointestinal tract.
(Figure 4C-F). The morphology and immunoprofile of the tumor strongly supported a diagnosis of MPNST.

After an uneventful postoperative course, the patient was discharged on the ninth postoperative day. At 12 months after surgery, the patient was in good condition, and no evidence of local recurrence or distant metastases was noted.

\section{Discussion}

MPNSTs are rare soft tissue tumors that arise in proximity to large peripheral nerves and account for $3-10 \%$ of

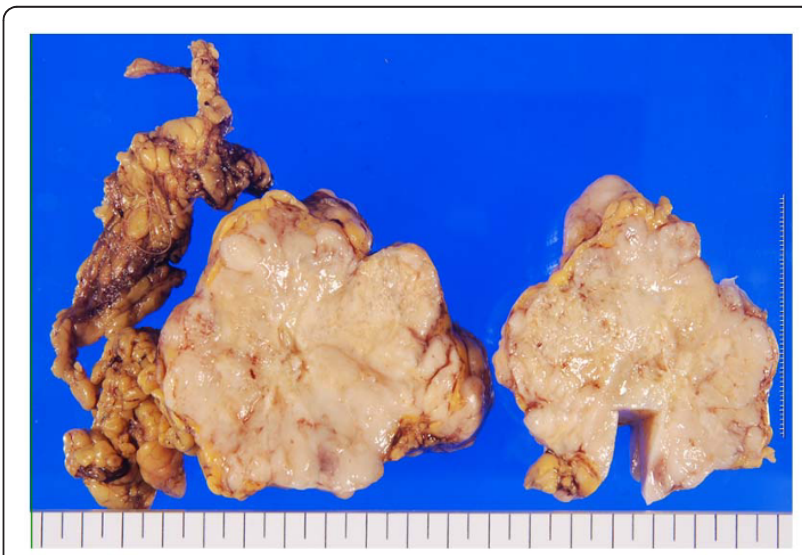

Figure 3 Macroscopically, the tumor is whitish grey and is relatively firm and solid. 

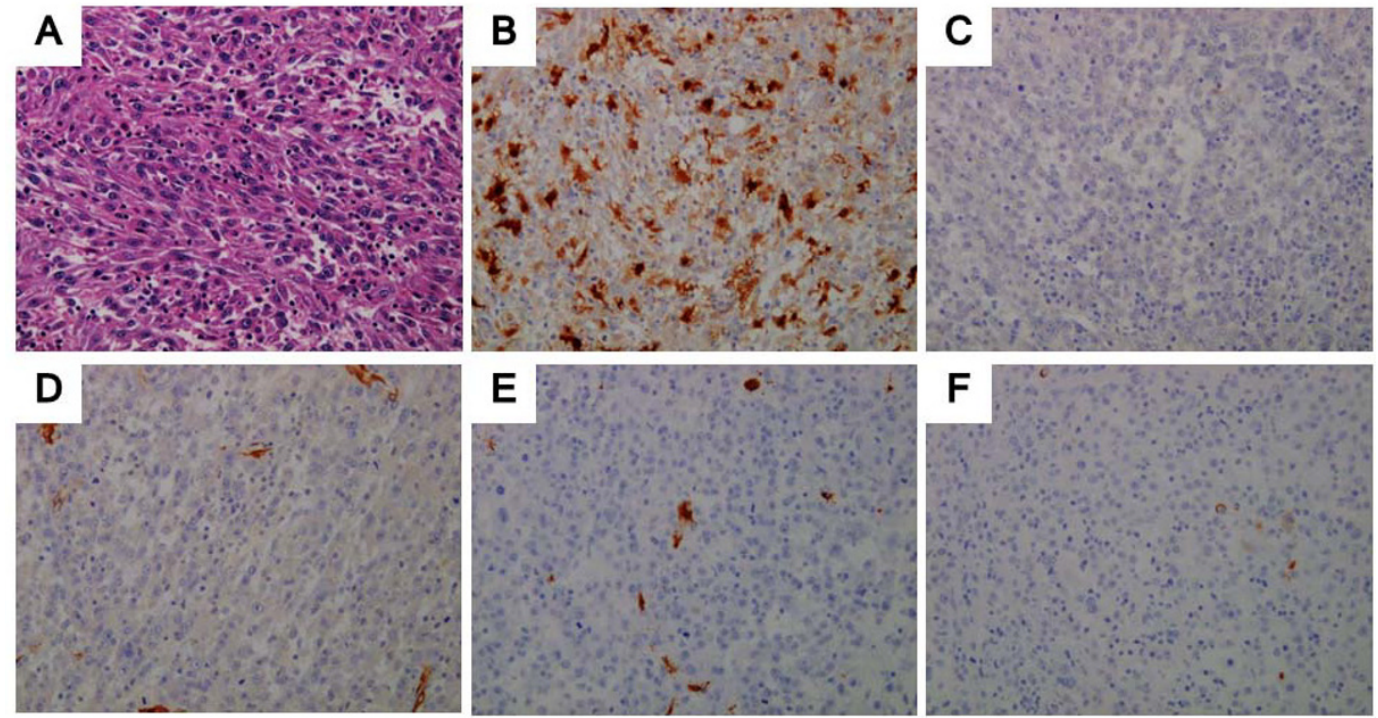

Figure 4 Microscopic analysis (A: hematoxylin-eosin (HE) stain; B, C, D, E, F: immunohistochemical analysis). A: Spindle cells arranged in intersecting fascicles and polygonal cells arranged in sheets grow infiltrating. The cellular nuclei are polygonal (bulky, roundish, and irregular), and the tumor cells show 150 mitoses per 50 high-power fields (HE stain; magnification, $\times 20$ ). B, C, D, E, F: Immunohistochemical images show positive staining of tumor cells for S-100 but negative staining for c-kit, $\alpha$-SMA, desmin, and CD34. (B: S-100, C: c-kit, D: $\alpha$-SMA, E: desmin, F: CD34)

all soft tissue sarcomas [2,3]. The term MPNST was coined by the World Health organization (WHO) and is defined as any tumor that arises from a peripheral nerve; this term replaces previously used heterogeneous and often confusing terminology, such as malignant schwannoma, malignant neurilemmoma, and neurofibrosarcoma, for tumors of neurogenic origin and similar biological behavior. These tumors arise from major or minor peripheral nerve branches or from the sheath of peripheral nerve fibers. Most of these tumors arise on the trunk, extremities, or the head and neck region $[4,5]$. MPNSTs arising from the abdominal cavity are extremely rare. Only a few cases of MPNSTs arising from the gastrointestinal tract have been reported [6,7], and to date, no cases of MPNSTs arising from the greater omentum have been reported in the literature. Although 4 cases of "benign schwannoma" of the greater/lesser omentum have been reported in earlier studies [8-11], high mitotic activity, which indicates malignant potential, was noted only in our patient. Therefore, to the best of our knowledge, this is the first reported case of an MPNST arising from the greater omentum.

The pathologic diagnosis of MPNST is facilitated by features such as palisading arrangement, nuclear atypia, bizarre giant cells, mitotic figures, and necrosis. These tumors have morphological heterogeneity, and staining analysis of such tumors reveals spindle cells with a fascicular pattern [12]. Histological and immunohistochemical markers specific for MPNSTs are not available. The
S100 protein is the antigen most commonly used to identify nerve sheath tumors of various types. However, S100 protein immunoreactivity is detected in only 50$60 \%$ of MPNSTs, and this protein is also expressed in a range of other tissues and tumor types [13,14]. Different markers are used to exclude other spindle cell tumors. Desmin and $\alpha$-SMA are used to exclude smooth muscle tumors, and CD34 and CD117 (c-kit) are used to exclude GIST [15]. In our case, the strong S-100 expression without expression of other immunohistochemical markers indicated the presence of an MPNST.

To date, little is known about MPNSTs arising from the abdominal cavity. Therefore, the prognosis of and initial treatments for such tumors are uncertain. A recently published study investigated the overall prognostic factors and survival of patients with MPNSTs in all locations $[4,5]$. The results of this study, which involved patients with localized MPNSTs, suggested that the disease-specific survival rate for MPNSTs was around $50 \%$ at 5 years. Most clinical series reported that tumor size was the most reliable independent prognostic factor; larger tumor size was related with worse outcome. Zou et al. reported that negative staining results for S-100 were associated with prognosis when the tumors were completely resected [5].

Survival appears to be related to complete tumor resection. Therefore, complete surgical resection of the tumor in patients with MPNSTs is of utmost importance for their treatment. 
It remains uncertain whether chemotherapy and radiotherapy have a positive impact on the survival of patients with MPNSTs. The results of most case series indicate limited benefits and high morbidity on using adjuvant radiotherapy or chemotherapy. Despite aggressive combined radiation and systemic chemotherapy, the 5 -year survival rates for MPNSTs range from 35\% to $50 \%[16,17]$. The current recommendation is that this therapy be reserved for recurrent tumors, suspected residual microscopic disease, and high-grade tumors [7].

Although these data may only describe what is known regarding the behavior of this tumor in other locations of the body, we recommend wide excision of MPNSTs with very close postoperative follow-up imaging.

\section{Conclusion}

MPNSTs arising from the greater omentum are extremely rare. It is important to recognize that an abdominal mass may be caused by an MPNST. MPNSTs should be considered as a rare differential diagnosis for a tumor in the greater omentum.

Because no definite microscopic criteria are available for distinguishing between benign and malignant tumors, radical excision is the treatment of choice for MPNSTs, and prolonged follow-up is essential.

\section{Consent}

Written informed consent was obtained from the patient for publication of this case report and any accompanying images. A copy of the written consent is available for review by the Editor-in-Chief of this journal

\section{Abbreviations}

CA: carbohydrate antigen; CEA: carcinoembryonic antigen; CT: computed tomography; FDG: fluorodeoxyglucose; GIST: gastrointestinal stromal tumor; MPNST: malignant peripheral nerve sheath tumor; PET: positron emission tomography; WHO: World Health Organization; a-SMA: a-smooth muscle actin.
\end{abstract}

\section{Authors' contributions}

MM participated in treatment of the patient, collected case details, literature search and draft the manuscript. YT participated in treatment of the patient and helped to draft the manuscript. HE, TH, TA, YK, MS, MT and MO participated in treatment of the patients. $\mathrm{HO}$ participated in treatment planning of the patient and helped to draft the manuscript. All authors read and approved the final manuscript.

\section{Competing interests}

The authors declare that they have no competing interests.

Received: 20 January 2011 Accepted: 21 March 2011

Published: 21 March 2011

\section{References}

1. Sompayrac SW, Mindelzun RE, Silverman PM, Sze R: The greater omentum. AJR Am J Roentgenol 1997, 168:683-687.

2. Pisters PW, Leung DH, Woodruff J, Shi W, Brennan MF: Analysis of prognostic factors in 1,041 patients with localized soft tissue sarcomas of the extremities. J Clin Oncol 1996, 14:1679-1689.
3. Stoeckle $E$, Coindre JM, Bonvalot $S$, Kantor $G$, Terrier $P$, Bonichon F, Nguyen Bui B: Prognostic factors in retroperitoneal sarcoma: a multivariate analysis of a series of 165 patients of the French Cancer Center Federation Sarcoma Group. Cancer 2001, 92:359-368.

4. Anghileri $M$, Miceli R, Fiore $M$, Mariani $L$, Ferrari A, Mussi $C$, Lozza $L$, Collini $P$, Olmi $P$, Casali $P G$, et al: Malignant peripheral nerve sheath tumors: prognostic factors and survival in a series of patients treated at a single institution. Cancer 2006, 107:1065-1074.

5. Zou C, Smith KD, Liu J, Lahat G, Myers S, Wang WL, Zhang W, McCutcheon IE, Slopis JM, Lazar AJ, et al: Clinical, pathological, and molecular variables predictive of malignant peripheral nerve sheath tumor outcome. Ann Surg 2009, 249:1014-1022.

6. Nozu T, Takahashi A, Asakawa H, Uehara A, Kohogo Y, Suzuki T: Malignant intestinal schwannoma: a case report and a review of the literature in Japan. Intern Med 1995, 34:1101-1105.

7. Telem DA, Pertsemlidis D: Malignant peripheral nerve sheath tumor: an unusual cause of intussusception. J Gastrointest Surg 2008, 12:1609-1611.

8. Bankier AA, Stanek C, Hubsch P: Case report: benign solitary schwannoma of the greater omentum: a rare cause of acute intraperitoneal bleedingdiagnosis by CT. Clin Radiol 1996, 51:517-518.

9. Sakai F, Sone S, Yanagisawa S, Ishii Z: Schwannoma of the lesser omentum. Eur J Radiol 1988, 8:113-114.

10. Tanoue Y, Tanaka N, Nagai M, Suzuki Y: Benign Pigmented Schwannoma of the Great Omentum: Report of a Rare Case and Review of the Literature. Case Rep Gastroenterol 2009, 3:222-229.

11. Totterman S, Lindfors $O$, Nickels J: A schwannoma of the lesser omentum. Rofo 1980, 132:585-586.

12. Enzinger FM, Weiss SW, (Eds): Malignant Tumors of Peripheral Nerves. St Louis (MO): Mosby; 31995.

13. Gonzalez-Martinez T, Perez-Pinera P, Diaz-Esnal B, Vega JA: S-100 proteins in the human peripheral nervous system. Microsc Res Tech 2003, 60:633-638.

14. Klijanienko J, Caillaud JM, Lagace R, Vielh P: Cytohistologic correlations of 24 malignant peripheral nerve sheath tumor (MPNST) in 17 patients: the Institut Curie experience. Diagn Cytopathol 2002, 27:103-108.

15. Kim JG, Sung WJ, Kim DH, Kim YH, Sohn SK, Lee KB: Malignant peripheral nerve sheath tumor in neurofibromatosis type I: unusual presentation of intraabdominal or intrathoracic mass. Korean J Intern Med 2005, 20:100-104.

16. Carli M, Ferrari A, Mattke A, Zanetti I, Casanova M, Bisogno G, Cecchetto G, Alaggio R, De Sio L, Koscielniak E, et al: Pediatric malignant peripheral nerve sheath tumor: the Italian and German soft tissue sarcoma cooperative group. J Clin Oncol 2005, 23:8422-8430.

17. Ducatman BS, Scheithauer BW, Piepgras DG, Reiman HM, Ilstrup DM: Malignant peripheral nerve sheath tumors. A clinicopathologic study of 120 cases. Cancer 1986, 57:2006-2021.

doi:10.1186/1477-7819-9-33

Cite this article as: Miguchi et al:: Malignant peripheral nerve sheath tumor arising from the greater omentum: Case report. World Journal of Surgical Oncology 2011 9:33.

\section{Submit your next manuscript to BioMed Central and take full advantage of:}

- Convenient online submission

- Thorough peer review

- No space constraints or color figure charges

- Immediate publication on acceptance

- Inclusion in PubMed, CAS, Scopus and Google Scholar

- Research which is freely available for redistribution 